\title{
Norois
}

Environnement, aménagement, société

$221 \mid 2011$

Innovations et agricultures urbaines durables

\section{Consommation d'espace agricole et relations entre acteurs privés et publics : un management en faveur de l'artificialisation}

Farm land consumption and relationships between private and public actors: a negotiation in favour of artificial land

José Serrano et Gisèle Vianey

\section{(2) OpenEdition}

Journals

Édition électronique

URL : http://journals.openedition.org/norois/3799

DOI : $10.4000 /$ norois.3799

ISBN : 978-2-7535-1838-4

ISSN : $1760-8546$

Éditeur

Presses universitaires de Rennes

Édition imprimée

Date de publication : 30 décembre 2011

Pagination : 111-124

ISBN : 978-2-7535-1786-8

ISSN : 0029-182X

Référence électronique

José Serrano et Gisèle Vianey, «Consommation d'espace agricole et relations entre acteurs privés et publics : un management en faveur de l'artificialisation », Norois [En ligne], 221 | 2011, mis en ligne le 30 décembre 2013, consulté le 20 avril 2019. URL : http://journals.openedition.org/norois/3799 ; DOI : 10.4000/norois.3799 


\title{
CONSOMMATION D'ESPACE AGRICOLE ET RELATIONS ENTRE ACTEURS PRIVÉS ET PUBLICS : \\ UN MANAGEMENT EN FAVEUR DE L'ARTIFICIALISATION
}

\author{
José Serrano \\ CITERES - UMR 6173 CNRS \\ (Université de Tours), \\ MSH Villes et territoires \\ 33 allée Ferdinand-de-Lesseps, BP 60449 - 37204 Touns cedex 03, France \\ jose.serrano@univ-tours.fr \\ GisÈLE VIANEY \\ Géographe-experte \\ 1888 route de la Frasse - 73200 Mercury, France \\ gisele.vianey@wanadoo.fr
}

\section{RÉSUMÉ}

À partir de l'examen de l'usage de l'espace, l'objectif de l'article est d'analyser une politique de développement local dans les espaces périurbains. L'usage des sols y fait l'objet d'une concurrence intense entre les différents acteurs économiques. Les élus locaux à travers le contrôle de l'affectation des sols sont en position d'arbitrer. L'article est centré sur les relations entre les acteurs économiques privés et les élus locaux. Allant à l'encontre des préconisations du développement durable, il ressort que les élus encouragent la consommation d'espace. Ils considèrent l'espace agricole comme une réserve foncière pour répondre aux acteurs privés et négligent la fonction de production alimentaire.

MotS CLÉ : consommation d'espace - zone d'activités - gestion de l'espace - espace périurbain

\section{ABSTRACT}

Farm land consumption and relationships between private and public actors: a negotiation in favour of artificial land

The aim of the paper is to analyze a local development policy through land use. In the periurban areas, land use is intensely fought over by economic actors. Local elected people can make decisions about the final use through their urban planning documents. The article focuses on the relationships between public and private actors. Going against the principles of sustainable development, local elected people are increasing soil consumption. They consider agricultural areas as land stock and they use it to respond to private interest. They neglect food production.

KEYWORDS : soil consumption - business park - land use management-periurban area 
Dans les communes périurbaines, les habitants bénéficient souvent de logements individuels, avec espaces verts, ainsi que d'équipements en services et commerces quotidiens (Roux et Vanier, 2008). En revanche, la dotation de ces communes en équipements de niveau moyen est faible. Ce déficit est compensé par une très bonne accessibilité aux grands centres commerciaux et aux zones d'activités situées en périphérie de l'agglomération (INRA et INSEE, 1988) et à proximité des échangeurs autoroutiers et des rocades. La surface agricole consommée pour la réalisation de routes et de parkings témoigne de l'amélioration continue de l'accessibilité routière à ces espaces (Pointereau et Coulon, 2009) où les disponibilités foncières demeurent importantes (Roux et Vanier, 2008). Et, si l'agglomération centre continue de concentrer les emplois, pour leur développement, les établissements consommateurs d'espace se délocalisent vers les communes périurbaines offrant disponibilité foncière et facilités de communications.

En dotant les collectivités locales de droits et de compétences précises, la décentralisation permet aux élus locaux d'élaborer et de conduire des stratégies de développement local (Bonnard, 2009). Par le renforcement de leurs missions, les communes ont la compétence pour prendre seules les décisions publiques concernant leur espace. Habilitées en matière d'aménagement et d'urbanisme, elles établissent les Plans Locaux d'Urbanisme (PLU), moyens d'instruire les demandes d'occupation des sols et d'élaborer un projet général d'aménagement tout en intégrant les questions de déplacement, de logement etc. Le rapprochement des décisions d'aménagement des échelons locaux devrait se traduire par une contextualisation des projets à savoir, une prise en compte du caractère hybride de l'espace périurbain. Lors de la formulation des projets d'aménagement, les élus ont à concilier entre les exigences consécutives des évolutions économiques concernant l'ensemble des activités et les représentations que les résidents et les agriculteurs ont des activités agricoles, de leur présent et de leur devenir.

Dans une campagne urbanisée, lorsqu'aux différents niveaux de la décision, l'ensemble des activités agricoles est convoqué, une dimension territoriale est introduite. Posant la question de l'alternative entre croissance économique et processus de développement, elle met la ressource foncière des espaces ouverts à la confluence des politiques agricoles et des politiques d'aménagement; cette dimension territoriale permet d'envisager de créer des interfaces et de modifier la politique foncière agricole qualifiée « à part » par Coulomb (1999). A contrario, lorsque dans des communes périurbaines, les espaces agricoles sont considérés comme des réserves foncières, la décision vise à satisfaire la demande individuelle croissante d'espace et le plan de relance des grandes infrastructures de transport et de la construction, en réponse à la crise. Il n'est alors pas tenté d'infléchir la tendance à l'artificialisation (Pointereau et Coulon, 2009).

Dans cet article, nous faisons l'hypothèse que la préservation des sols agricoles dépend de la valeur attribuée à ces espaces par les agents économiques qui les utilisent et des rapports de forces entre ces agents. Pour étayer cette hypothèse nous procédons à l'examen de la mise à disposition de foncier par la communauté de communes du Vouvrillon. Les résultats reposent sur deux séries d'entretiens semi-directifs. La première, menée auprès des collectivités locales incluses dans l'aire du SCoT de l'agglomération tourangelle vise à montrer les liens entre les stratégies de développement économique des élus locaux et la recherche de nouvelles ressources fiscales. La seconde série se focalise sur le cas de l'une des communautés de communes tourangelles qui initie une opération d'aménagement d'une zone économique ${ }^{1}$. Elle s'attache à caractériser les rapports entre acteurs publics et privés au moment de l'élaboration des décisions. Les relations entre acteurs publics et privés ont été peu abordées dans la littérature scientifique et constituent l'originalité du propos.

Notre analyse se réfère principalement aux champs théoriques de la géographie sociale qui permet de saisir les interactions entre les éléments de l'espace; cette géographie considère l'espace informé par le fait social et le traite en ayant recours aux apports et aux méthodes de disciplines humaines et sociales connexes (Chivallon, 2004). Pour appréhender comment l'espace est organisé, relier les discours sur l'espace aux objets spatiaux, saisir les relations que les hommes

1. Ce travail a été mené en 2006 avec Elen Cornec étudiante en Master recherche «Villes et territoires »-université de Tours. 
entretiennent entre eux sur des bases diverses (sociologique, économique, culturelle, etc.), ce courant disciplinaire postule que les faits sociaux ont par leur localisation et manifestation des dimensions spatiales et, inversement, que les faits géographiques, de par leur dimension spatiale, comportent des aspects sociaux (Raffestin, 1986; Del Casino et al., 2000). Notre propos est enrichi par la théorie des représentations et des pratiques sociales (Abric, 2003; Rouquette et Rateau, 1998) : emprunt à la psychologie sociale, cette théorie permet à la géographie d'associer une analyse des réalités idéelles à celle des réalités matérielles. La théorie des représentations et des pratiques sociales (Abric, 2003; Rouquette et Rateau, 1998) et celle de la construction de l'accord (Moscovici et Doise, 1992) sont les moyens de saisir les significations et les valeurs attribuées à l'espace, et de prendre en charge la construction de sens autour de la question de la place de l'activité agricole. Ces théories caractérisent les représentations sociales par deux traits complémentaires : leur historicité (produits de l'histoire, elles participent à l'histoire) et leur altérité, c'est-à-dire le fait qu'elles adviennent toujours dans le cadre de relations intergroupes. Les représentations sociales sont à la fois organisatrices de l'expérience, régulatrices de la conduite et pourvoyeuses de valeurs (Abric, 2003; Rouquette et Rateau, 1998). Ce corpus disciplinaire nous permet de saisir l'inscription spatiale des faits sociaux et des actions politiques (Santos, 1990).

Dans la première partie, nous montrons comment les élus locaux utilisent le foncier comme moyen de développement privilégié en conjuguant attractivité des espaces périurbains et abondance des réserves foncières. La valorisation du sol comme moyen de développement entraine des changements d'usages. Dans la seconde partie, nous présentons l'influence des acteurs privés sur les décisions prises par les élus. Avant de conclure, la troisième partie aborde les conséquences de cette voie de développement pour l'aménagement du front urbain.

\section{Le foncier, un moyen de développement privilégié dans des espaces attractifs}

\section{LA MISE À DISPOSITION DE FONCIER POUR ALIMENTER LA COMPÉTITION ENTRE LES COLLECTIVITÉS LOCALES}

Dans l'aire étudiée, la coopération intercommunale est structurée autour de quatre communautés de communes et d'une communauté d'agglomération créées en 2000 et $2001^{2}$. L'engagement des élus dans les regroupements intercommunaux est le moyen de financer, pour la population, des services ou des équipements supplémentaires ${ }^{3}$. Le financement de ces nouveaux services (petite enfance, équipements sportifs conséquents, etc.) nécessite de dégager des ressources fiscales propres complémentaires ce qui alourdit la fiscalité sur les ménages, sur les entreprises ou sur les deux. Les cinq groupements intercommunaux ont choisi d'épargner les ménages et de recourir à la taxe professionnelle unique. En faisant peser la croissance de leur budget sur l'accueil exclusif de nouvelles entreprises, les élus lient le financement de l'action intercommunale à la conjoncture économique. Cette taxe est perçue en fonction du lieu d'implantation des entreprises. De cette compétition entre élus pour capter la taxe professionnelle résultent de grandes disparités entre les communes (fig. 1).

$70 \%$ des emplois de l'aire urbaine relèvent du tertiaire. La répartition spatiale des emplois montre une organisation concentrique et une concentration au noyau urbain. Le centre est le siège des services et de l'industrie des biens de consommation. Les industries de biens d'équipement sont plus nombreuses en banlieue et les entreprises de construction sont surreprésentées dans le périurbain (DRE région Centre et al., 2001). Entre 1998 et 2006, l'aire du SCoT de Tours dans lequel est insérée la communauté de communes du Vouvrillon a connu une progression de $10 \%$ des établissements et de $4 \%$ des emplois. Les services à la personne, les services opération-

2. Depuis le $1^{\text {er }}$ janvier 2009, le paysage institutionnel local a évolué. La communauté de communes de la confluence a intégré la communauté d'agglomération de Tours $(+)$.

3. Enquêtes élus. 


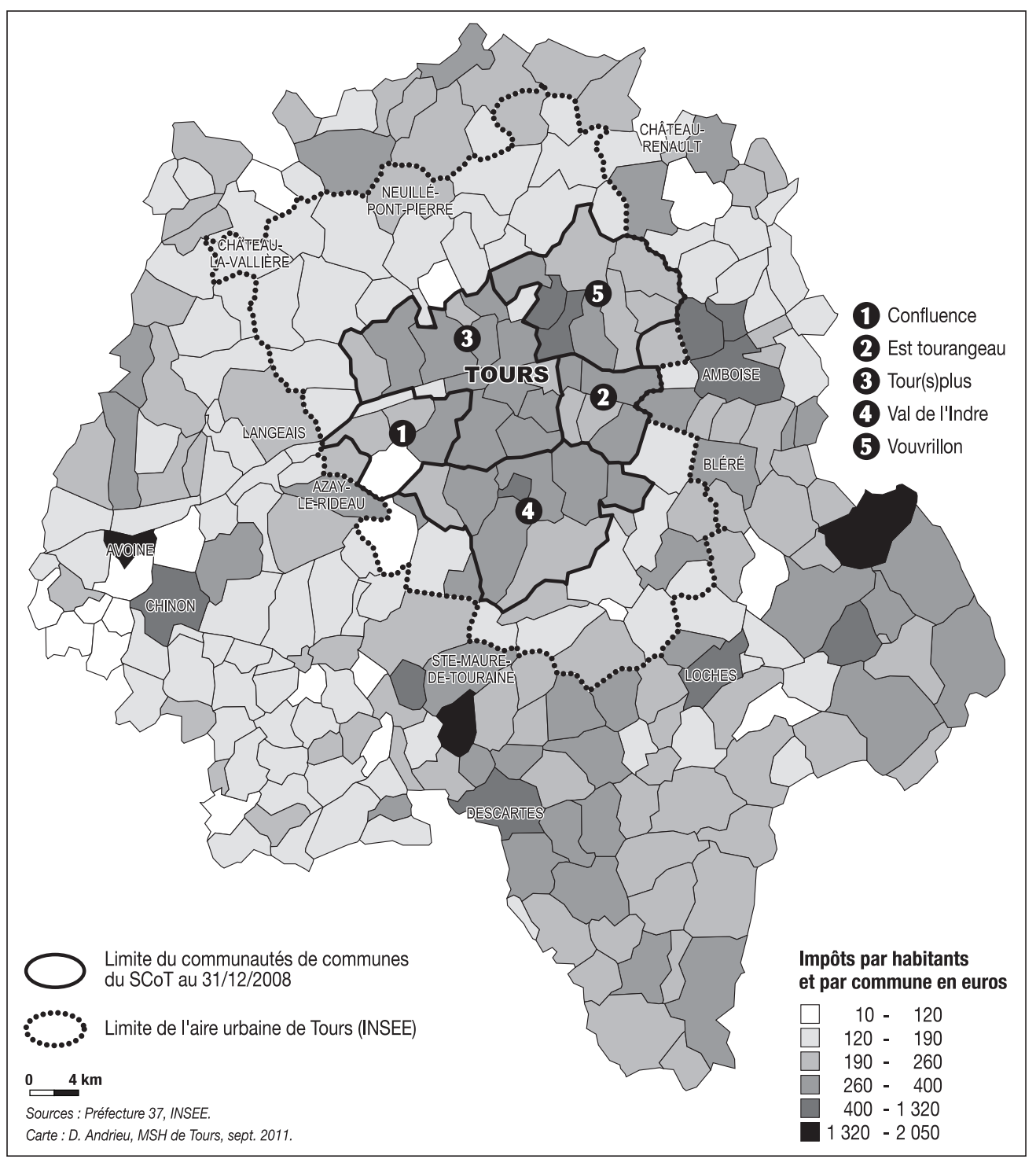

Figure 1 : Produits des impôts locaux par habitants en 2000 en Indre-et-Loire Profit of local taxes per inhabitants in 2000 in Indre-et-Loire

nels et la construction sont les secteurs les plus croissants. Dans une moindre mesure, les services collectifs et de transport, la logistique et le commerce de gros ont également progressé (Agence d'urbanisme de l'agglomération tourangelle, 2006).

Spatialement, on observe un mouvement centrifuge des activités mais le poids économique du noyau urbain reste prépondérant (tableau 1). Les services aux entreprises, supérieurs et opérationnels, - en forte croissance -, continuent de se concentrer dans le noyau urbain. La logistique et le transport, faits de grands groupes internationaux sont en très fort développement et nécessitent d'importantes disponibilités foncières. La construction, en expansion, est constituée de petites et moyennes entreprises dispersées dans l'ensemble du périurbain. 


\begin{tabular}{|c|c|c|c|}
\hline Branche d'activité & Degré de concentration & $\begin{array}{c}\text { Degré d'agglomération } \\
\text { spatiale }\end{array}$ & Fort \\
\hline $\begin{array}{c}\text { Services : aux entreprises, } \\
\text { supérieurs et opérationnels }\end{array}$ & PME & Faible & $\begin{array}{c}\text { Localisation } \\
\text { d'activité }\end{array}$ \\
\hline $\begin{array}{c}\text { Production industrielle } \\
\text { Transport et logistique }\end{array}$ & $\begin{array}{c}\text { Grands établissements : } \\
\text { groupes nationaux ou } \\
\text { internationaux }\end{array}$ & Faible & $\begin{array}{c}\text { Polarisation dans les zones } \\
\text { d'activités }\end{array}$ \\
\hline Construction & PME & Dispersion \\
\hline
\end{tabular}

Tableau 1 : Localisation et organisation spatiale des différentes branches économiques (source : Serrano d'après Agence d'urbanisme de Tours, 2006)

Location and spatial organisation of economic sectors

Dans l'aire du SCoT de Tours, les différents Établissements Publics de Coopération Intercommunale (EPCI) convoitent tous le même segment : la logistique. Ce choix s'explique par le dynamisme du secteur et par l'ouverture concomitante de nouvelles infrastructures autoroutières : ouverture des autoroutes A28 et A85 qui coupent toutes les deux l'A10 et prolongement du périphérique qui améliore leurs connections. Chaque EPCI a sur son territoire un croisement d'autoroutes ou une sortie d'autoroute (fig. 2); tous misent sur ces nouvelles opportunités et se livrent une course pour réaliser et remplir leurs zones d'activités respectives. La communauté d'agglomération de Tours, dont la politique de développement est plus diversifiée dispute l'installation d'activités logistiques aux communautés voisines. Seule la communauté de communes de l'est tourangeau n'a pas d'autoroutes sur son territoire : elle conduit une politique de développement originale en soutenant économiquement la filière viticole et en développant un programme de bureaux.

Pour leur développement économique, les communautés de communes cherchent à mettre à disposition du foncier équipé : c'est le cas de la communauté de communes du Vouvrillon. La santé économique des entreprises dépend de leurs stratégies commerciales. En limitant leur action économique à une offre de surfaces, les communautés de communes se mettent en forte concurrence. De plus, mobilisant les mêmes outils pour offrir les mêmes ressources aux entreprises, toutes se focalisent sur le même segment économique. En démarchant des entreprises et en cherchant à conforter leur ancrage local en promouvant des collaborations avec des laboratoires de recherches universitaires locaux, la communauté d'agglomération fait exception.

\section{LE VOUVRILLON : UN SECTEUR OFFRANT DES SOLS À TRÈS FORT POTENTIEL ET D'IMPORTANTES OPPORTUNITÉS DE DÉVELOPPEMENT ÉCONOMIQUE}

La communauté de communes du Vouvrillon est à une dizaine de kilomètres au nord-est de Tours. Son paysage, un plateau agricole ondulé et ponctué de bosquets est entaillé par des vallées encaissées. Un vignoble classé en appellation Vouvray occupe le sud et l'est du plateau. Les bourgs sont ramassés mais les extensions récentes suivent les axes routiers. Cette communauté de communes embrasse deux petites régions agricoles : le vignoble de Vouvray et la Gâtine tourangelle occupée par une polyculture à dominante céréalière évoluant vers la monoculture céréalière. Dans cette région agricole, les agriculteurs, à l'initiative d'opérations de remembrement, de drainage des sols bruns et d’irrigation, améliorent le foncier. Au sud de la Gâtine tourangelle, le plateau de Mettray a été retenu pour implanter la zone d'activités du Cassentin. Ce plateau aux très bons sols calcimagnésiques est propice à la céréaliculture (DDAF 37, 2005). La deuxième production marquante est le vignoble de Vouvray : six des huit communes de la communauté de communes sont dans l'aire d'appellation. Grâce à une politique d'amélioration de la qualité, le vignoble est en expansion. La production est majoritairement écoulée par le négoce bien que les vignerons développent de plus en plus la vente directe en bouteille (Ministère de l'agriculture et de la pèche, 2001). 


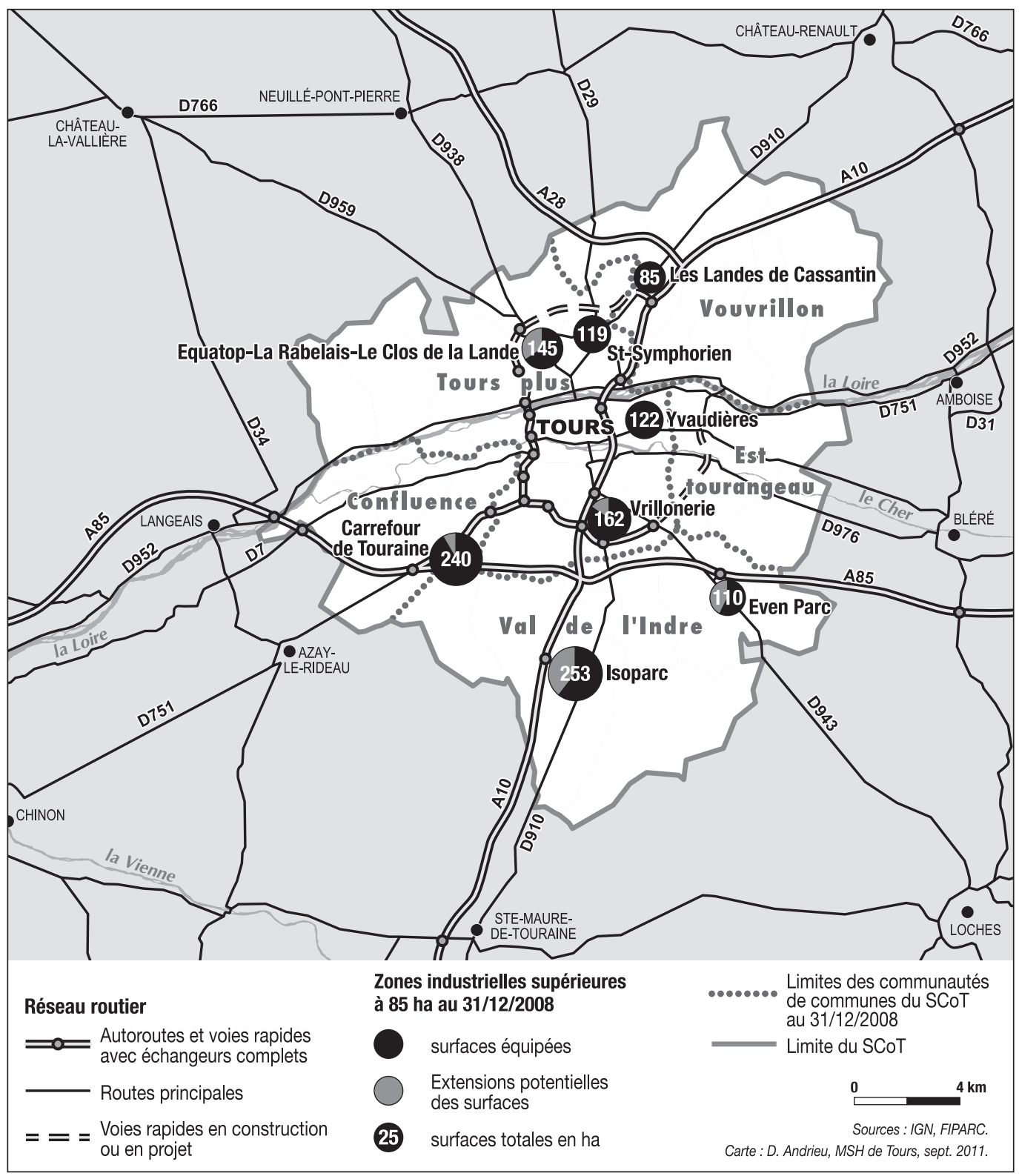

Figure 2 : Les zones industrielles du SCoT de Tours au 31 décembre 2008

The business parks in the SCoT (regional coherence plan) of Tours at december $31^{\text {st }} 2008$

C'est précisément ce plateau qui est désigné comme site majeur de développement économique au sein de l'aire du SCoT de Tours (Agence, d'urbanisme de l'agglomération tourangelle, 2006). Il cumule l'intersection de deux autoroutes à vocation internationales et des zones d'activités orientées vers la logistique et le transport routier (fig. 2). En 2002, la communauté de communes du Vouvrillon décide d'implanter une nouvelle zone d'activités de 90 ha en prolongement de la zone des «landes du Cassentin » (fig. 2). Concomitamment, la commune de Vouvray révise son 
plan local d'urbanisme. Ces opérations ne sont pas menées à huis clos par les municipalités. Les élus locaux sont en lien avec les acteurs économiques utilisateurs du foncier qui font valoir leurs intérêts propres. Les choix opérés au cours de la révision du document d'urbanisme et la manière dont sont menées les évictions agricoles nécessaires à la réalisation de la zone d'activités révèlent la valeur attribuée aux différents usages des sols.

Dans le cadre de l'élaboration du SCoT de Tours, le diagnostic économique de la communauté de communes du Vouvrillon relève une augmentation de $26 \%$ des emplois entre 1999 et 2003, un taux de chômage inférieur à la moyenne nationale : 7,6 \% contre 8,4 \% en 2005. Les zones d'activités accueillent surtout de grands établissements (16\% des établissements mais $50 \%$ des emplois) et fournissent $46 \%$ de la taxe professionnelle unique. Les activités de construction sont légèrement surreprésentées par rapport à la moyenne départementale. L'essentiel de l'activité économique est localisée en dehors des zones d'activités. Pourtant, c'est sur elles que la communauté de communes centre son intervention; alors que 67 des 228 ha de zones d'activités qu'elle possédait en 2006 sont inoccupés, elle prévoit l'ouverture de 160 ha. Le tissu économique est diversifié mais deux filières de renommées internationales sont mises en exergue par les élus locaux : la logistique et la viticulture. Des entreprises de logistique (Norbert Dentressangle, Géodis Dusolier Calberson, TFE, etc.) ont implanté des établissements de plusieurs centaines de salariés. Les 250 petites structures viticoles emploient 300 salariés.

En retenant d'accueillir les entreprises sur les très bons sols calcimagnésiques du plateau, il est implicitement procédé à une hiérarchie des valeurs attribuées aux espaces agricoles.

\section{Les espaces agricoles définis en fonction de leurs valeurs économiques}

\section{Des COMMUNeS SOUS LA PRESSION DES LOBBYS}

\section{L'entreprise de transport}

La zone d'activités du Cassentin couvre 90 ha; elle prolonge celle des landes du Cassentin et vise à agrandir l'emprise de l'entreprise Norbert Dentressangle occupant déjà sept hectares. Le projet impacte trois exploitations agricoles.

Sur cette zone, le projet d'un centre régional routier date des années 1970. Abandonné et retiré du schéma directeur d'aménagement de Tours en 1995 suite à l'échec de Jean Royer aux élections municipales, il est remis à l'ordre du jour en 2002 par la communauté de communes du Vouvrillon. La majorité de l'espace intercommunal est occupé par des vignobles. Seul le plateau du Cassentin, à proximité des infrastructures autoroutières, est consacré aux grandes cultures. L'entreprise Norbert Dentressangle, second transporteur routier français, emploie localement 300 personnes. Depuis plusieurs années, l'entreprise sollicite l'intercommunalité; son activité croissante génère plus de 400 entrées et sorties de poids lourds sur la départementale 910 et cause des problèmes de circulation et de sécurité. En décembre 2003, l’action du PDG précipite le projet : accompagné d'élus régionaux, il fait une descente en hélicoptère sur le site et menace de se délocaliser. Par cette pression, la communauté de communes valide une première tranche de 50 ha de la zone d'activités. Elle informe la DDAF et la chambre d'agriculture d'un projet de zone d'activités. La communauté de communes justifie le projet par son inscription « dans la politique globale de développement économique de la communauté de communes. Il vient compléter la gamme du parc d'activités du Cassentin, spécialisé en transport et logistique [...] Il représente de fait une opportunité de développement et de création d'emplois en réponse à la pénurie de foncier à vocation économique ». Pour la communauté, ce projet répond bien à la définition de l'intérêt général donnée par le ministère : "sont d'intérêt général, les projets d'équipement ou d'aménagement des collectivités publiques [...] comme l'implantation d'une activité créatrice d'emplois ». La stratégie des élus est confortée. 


\section{Les viticulteurs}

Entre 2001 et 2006, la commune de Vouvray révise son PLU. À proximité de Tours, la commune a une croissance démographique modérée : +4,61 \% entre 1990 et 1999. Elle souhaite simultanément poursuivre son développement, préserver son cadre de vie et garder son caractère rural.

Le vignoble occupe 2000 hectares dans l'aire d'appellation et génère 300 emplois directs dans 200 exploitations : c'est une économie locale importante. Des zones $\mathrm{Ux}^{4}$ pour le stockage des productions viticoles sont dessinées. Les plateaux non viticoles sont des réserves foncières pour les futurs aménagements, notamment l'extension, sur 7 ha, de la zone d'activités communale. Concertés pour la définition des zones du PLU, les agriculteurs entérinent la préservation du vignoble et l'ouverture à l'urbanisation des terres non viticoles. Dans le conseil municipal de Vouvray, les viticulteurs sont fortement représentés : sur les 23 membres, cinq sont viticulteurs et deux sont propriétaires de vignoble.

Les interventions de la chambre d'agriculture, la DDAF et l'INAO, personnes publiques associés à la révision du PLU, visent à éviter que les terres consommées par les aménagements soient celles classées en appellation et à conserver des espaces tampons afin de prévenir les problèmes de cohabitation entre activité agricole et résidents.

Le syndicat viticole de Vouvray a mis en place une commission constituée des sept représentants communaux de l'aire d'appellation. Il demande à être convié à la commission communale d'élaboration des documents d'urbanisme. Intégré à la réflexion, il veille à limiter le grignotage des terres viticoles. Il est garant de la pérennité de l'activité viticole en zone périurbaine. L'INAO estimant le syndicat des viticulteurs très présent dans l'élaboration des documents d'urbanisme n'émet pas de réserves quand le projet de PLU lui est présenté; présents et écoutés, les viticulteurs font inclure les problématiques viticoles dans le PLU.

\section{LE FONCIER AGRICOLE : MOYEN DE L’EXPANSION DE LA VILLE}

\section{Des élus peu concernés par l'agriculture}

Pour les élus de la communauté de communes du Vouvrillon, l'activité agricole ne relève pas d'une politique publique locale. Ils se disent incompétents pour proposer aux agriculteurs des modifications de pratiques ou de nouvelles orientations de production et renvoient ces problèmes aux conseillers agricoles de la chambre d'agriculture ou au niveau de la politique agricole commune sur laquelle ils disent n'avoir aucune prise. De plus, le décalage entre le langage des élus et celui des agriculteurs rend le dialogue encore plus difficile. Là où les élus parlent d'entretien de l'espace, de préservation de paysages identitaires, les agriculteurs parlent de production alimentaire et de marchés. Enfin, l'agriculture apparaît comme une activité économique en déclin. D'une part, les élus notent un recul du nombre d'exploitations. D'autre part, en amalgamant les jachères à des friches, ils déduisent que le secteur est en difficulté et qu'il ne peut plus occuper tout l'espace. Par contre, la production de Vouvray est en expansion. Cas particulier, elle fait figure d'exemple.

Dans le débat, la chambre d'agriculture ne s'oppose pas au développement économique et résidentiel, ce qui revient à ce qu’elle ne s'engage pas, en tant que représentant de la profession agricole, dans la défense du foncier agricole. Elle considère l'accueil de nouveaux habitants ou de nouvelles entreprises comme une richesse, qu'elle ne peut contrarier car elle n'en a pas la légitimité. La consommation d'espace consécutive à l'urbanisation est inéluctable. En réponse, elle préconise de créer des réserves foncières permettant aux agriculteurs affectés par un aménagement de reconstituer rapidement l'assise foncière de leur exploitation ${ }^{5}$. De fait, elle entérine, la délocalisation des exploitations; elle travaille également à l'élaboration d'un protocole d'indem-

\footnotetext{
4. Zone Ux : destinée à l'implantation d'établissements qui, en raison de la nature ou de l'importance de leurs activités ont plutôt leur place à l'écart des zones d'habitation mixte

5. Lettres du 6 avril 2006 au maire d'Artannes-sur-Indre et du 23 juillet 2006 au maire de Joué-lès-Tours.
} 
nisation facilitant la défense des intérêts des agriculteurs face à un maître d'œuvre. La chambre d'agriculture constate que les forêts et les vignobles sont plus épargnés par l'artificialisation grâce notamment à l'action l'INAO. Elle choisit d'accepter les aménagements et de se concentrer sur des mesures de remédiation foncière.

\section{La difficile prise en compte du maraîchage}

Pour la réalisation de la zone d'activités du Cassentin, la communauté de communes du Vouvrillon mandate la société d'équipement de Touraine et la charge d'effectuer les relevés et de mener les négociations pour les évictions des agriculteurs. Les trois exploitations agricoles impactées réagissent différemment (tableau 2).

\begin{tabular}{|c|c|c|c|}
\hline Exploitation évincée & $\begin{array}{c}\text { Couleur } \\
\text { Expl. Céréalière }\left(^{*}\right)\end{array}$ & $\begin{array}{c}\text { La Pérauderie } \\
\text { Expl. Céréalière }\end{array}$ & $\begin{array}{c}\text { Les jardins de Meslay } \\
\text { Expl. Maraîchère }\end{array}$ \\
\hline Surface impactée & N. C $\left(^{* *}\right)$ & 34,39 ha & 3,91 ha \\
\hline Indemnisation à l'hectare & N. C & $3000 €$ & $58500 €$ \\
\hline Devenir de l'exploitation & Délocalisation & $\begin{array}{c}\text { Reconversion en base de } \\
\text { loisirs }\end{array}$ & Délocalisation à proximité \\
\hline
\end{tabular}

(*) : enquêtes, $\left.{ }^{* *}\right)$ : Non communiqué

Tableau 2 : Devenir des exploitations agricoles selon le type d'activité (source : Registre délibérations de la communauté de communes du Vouvrillon, séance 12 octobre 2005)

The outcome for farms according to type of activity

Installé depuis trois mois, l'exploitant de la ferme Couleur apprend fin 2004, - par son propriétaire contacté par la société d'équipement -, l'impact du projet sur ses terres. Ce céréalier a réalisé des améliorations foncières en drainant ses parcelles. Il fait trainer les négociations pour faire monter les enchères. Les indemnités perçues vont lui permettre de reconstituer son foncier plus au nord et de continuer son activité céréalière. Cet exploitant est dans la ligne défendue par la chambre d'agriculture : ne pas s'opposer à la consommation d'espace mais négocier au mieux son éviction pour pouvoir délocaliser l'exploitation dans les meilleures conditions.

L'exploitant de la Pérauderie est à la retraite. Il apprend le projet par des rumeurs. Il est contacté en 2003 par la société d'équipement pour discuter le rachat de ses terres : la collectivité veut rapidement construire la route pour la société Dentressangle. Depuis 2002, cet agriculteur travaille sur un projet de base de loisirs : il le présente en 2004 à la communauté de communes qui le juge sérieux et l'intègre à la zone d'activités. La révision simplifiée du plan d'occupation des sols permet de trouver un accord entre chaque partie. En échange de la permission de réaliser l'accès à la zone d'activités immédiatement et de l'acceptation des conditions de rachats de ses terres, l'exploitant obtient le classement de ses terres en terrains constructibles et le branchement aux réseaux : il peut transformer l'exploitation agricole en base de loisirs.

La prise en compte de l'activité des jardins de Meslay est celle qui pose le plus de problèmes. Cette exploitation maraîchère pratique la cueillette à la ferme; située le long de la RD 910 cette position assure le succès de l'activité de cueillette. Là aussi, l'exploitant apprend par hasard le projet de zone d'activités susceptible de le concerner. Le projet de zone d'activités l'impacte fortement : une route d'accès coupe l'exploitation en deux. L'exploitant prend les devants mais, entre décembre 2003 et mai 2005, il se heurte à l'indifférence des élus de la communauté et de la société d'équipement. La proposition d'échange foncier qui lui est faite en juillet 2004 est inadaptée. En mai 2005, un article sur le devenir de l'exploitation paraît dans le quotidien local. Suite à cette parution, les habitants s'inquiètent, auprès des élus, du devenir des jardins de Meslay et menacent de faire circuler des pétitions. La société d'équipement contacte alors l'exploitant pour trouver une solution de pérennisation de l'exploitation. La spécificité de l'exploitation maraîchère est prise au sérieux puisque l'arrangement proposé tient compte de l'investissement foncier 
José Serrano, Gisèle Vianey

propre aux exploitations maraîchères (tableau 2) et de la localisation particulière de l'exploitation. Toutefois, les surfaces en jeu sont faibles.

\section{Des choix de développement au service de l'étalement et non de l'hybridation du périurbain}

\section{L'ÉCHELLE DE VALEURS DES SOLS AGRICOLES POUR LES ÉLUS LOCAUX}

Avec les documents d'urbanisme, les élus décident de l'affectation des sols. Tous les sols ont un usage y compris les rares friches (certaines sont en attente de spéculation). Pour remplacer un usage par un autre et justifier leurs choix, les élus ont besoin d'une échelle de valeur; elle leur permet de justifier des usages à modifier et de ceux à préserver.

Le projet de zone d'activités a confronté les élus à la diversité des exploitations agricoles. Ils ont d'abord appliqué une vision duale de l'agriculture qu'ils ont dû reconsidérer. Le sort fait aux exploitations dépend du lien qu'elles entretiennent avec la ville. La ferme de la Pérauderie transformée en base de loisirs et les jardins de Meslay sont finalement intégrés au projet d'aménagement et peuvent rester sur place. Indemnisée, la ferme de Couleur peut se relocaliser et reconstituer son assise foncière plus au nord. Néanmoins, les tribulations des jardins de Meslay montrent que la reconnaissance de ce lien en tant que construit social ne va pas de soi. Évacuant la fonction de production, les élus prennent en compte la préservation du cadre de vie et les loisirs : cette vision vaut pour le vignoble alors que les exploitations céréalières peuvent être délocalisées. Initialement, les jardins de Meslay sont perçus comme les cultures céréalières. Bien que les organisations professionnelles agricoles aient attiré l'attention sur leurs spécificités, c'est la mobilisation des clients des Jardins de Meslay qui permet leur reconnaissance. Dans ce débat, les jardins de Meslay ont glissé de la catégorie production alimentaire à celle de fournisseur de loisirs et de liens. La ferme de la Pérauderie qui, sans trop de difficultés, intègre la zone d'activités est aussi, orientée vers les loisirs : dans sa mutation, elle abandonne toute activité de production et ne garde de l'exploitation agricole, que les bâtiments reconvertis dans l'accueil du public.

Le classement en appellation d'origine repose sur la singularité du lien entre le terroir et le travail des vignerons. Le vin est devenu l'emblème de la communauté de communes. A contrario, l'activité céréalière intensive est une production banale. Occupant de vastes espaces, générant peu d'emplois cette production standardisée est perçue polluante et obsédée par la recherche de rendements. Elle n'est le motif d'aucune fierté. Pour les élus, le cadre rural est un élément identitaire autant qu'un atout pour le développement. Dans un contexte où les communes ou les groupements sont en concurrence pour l'accueil de résidents et d'entreprises, les élus mettent en avant le paysage, l'espace agricole et naturel comme éléments valorisants du cadre de vie.

Le transporteur a obtenu ce qu'il voulait. Il a pu s'étendre tout en continuant à bénéficier d'un accès facile et sécurisé au carrefour autoroutier. Il a traité directement et pour son propre compte les réaménagements avec les élus locaux. Pour le transporteur, le sol est un simple support physique sur lequel il installe son entreprise. La valeur du site est due à l'accessibilité routière.

Les viticulteurs ont fait valoir leur spécificité et leurs intérêts particuliers. Ils se sont profondément démarqués du reste de la profession agricole. Ils n’ont pas utilisé les organisations professionnelles agricoles départementales pour se défendre. Dotés d'un syndicat et d'organisations professionnelles agricoles spécifiques (INAO), ils ont défendu le foncier à l'intérieur du périmètre de l'appellation. Ils n’ont ni porté la défense des sols agricoles en général ni soutenu les autres types d'agricultures.

Les agriculteurs impactés par la zone d'activité ont réglé individuellement l'adaptation de leur système d'exploitation. Dans un contexte de baisse du nombre d'exploitations agricoles, le céréalier n’a pas eu de mal à reconstituer son assise foncière en se délocalisant de quelques kilomètres. L'exploitant des Jardins de Meslay, quant à lui, s'est trouvé bien seul lorsqu'il a s'agit de défendre le maintien de son activité sur place. Seule la mobilisation inopinée de sa clientèle lui a permis d'obtenir l'écoute des élus. 
Dans la conduite du projet d'aménagement, la disjonction de la fonction de production avec celle de fourniture d'autres services confirme que les élus ont une vision monofonctionnelle de l'agriculture (Duvernoy, 2002). Production de masse, circuits courts, production identitaire; cette vision est hiérarchisée. Les autres usages économiques s’insèrent dans cette échelle. Le vignoble du fait de sa spécificité est irremplaçable; il est sanctuarisé et placé en haut de l'échelle. Viennent ensuite les activités économiques non agricoles qui apportent recettes fiscales et emplois. Les circuits courts répondant à une demande sociale locale peuvent être prises en compte. Enfin, la production alimentaire de masse est considérée comme le plus bas niveau d'utilisation des espaces agricoles. C'est une activité par défaut.

Cette hiérarchie est possible car le foncier est abondant. Il n'y a pas de rareté qui oblige à poser l'alternative entre le vignoble et le développement économique. Or, cette rareté ne renvoie pas qu'à la valeur monétaire du foncier mais aussi au regard porté sur les usages du sol. Le mutisme de la profession agricole face à la protection des sols pour une production alimentaire de masse autorise les élus à établir cette hiérarchie. Un mutisme qui finalement arrange tout le monde : les viticulteurs peuvent déplacer la pression foncière en dehors de leurs terroirs, les entrepreneurs trouvent un foncier viabilisé assez facilement, les propriétaires réalisent des plus-values mais aussi les exploitants se délocalisent sans trop de mal grâce à de bonnes indemnités. De fait, la concurrence ne s'exprime pas. Les difficultés des jardins de Meslay montrent que reconsidérer la valeur d'une agriculture alimentaire fut-elle de proximité est dérangeant.

L'échelon communal est la scène de tensions, de négociations et de décisions des usages de l'espace. Le foncier agricole est au cœur de ces débats. En ouvrant des espaces de débats favorables à la résolution des conflits (Martin et al., 2006), l'élaboration des PLU est l'occasion de coordination entre acteurs. Le cas de la zone d'activité du Cassentin montre les arrangements opérés entre les acteurs économiques privés et les élus. À l'exception des vignobles, l'espace agricole est considéré comme une réserve foncière. Assez facilement, un consensus est trouvé entre acteurs privés et élus pour localiser les nouvelles activités économiques. Ce consensus est d'autant plus aisé que la vision de réserve n’est pas remise en question à une échelle trop grande.

\section{Préserver une agriculture Productive n’entre PAS DANS LES ENJEUX LOCAUX}

Le sol agricole peut être considéré comme une ressource non renouvelable. Sa préservation relève de l'intérêt général. En l'absence d'une profession agricole mobilisée sur le sujet, les collectivités locales pourraient au nom de l'intérêt général préserver les sols agricoles de l'artificialisation. Or, il n'en est rien.

Les stratégies adoptées par les collectivités révèlent une vision de l’intérêt général subordonnée à l'échelle locale y compris lorsqu'il s'agit de préserver les sols pour des raisons patrimoniales. Les communes périurbaines sont regroupées dans de jeunes intercommunalités. Leur légitimité est encore à asseoir dans leurs actions et dans leurs périmètres. Construites souvent en opposition à une agglomération, le cadre rural et le paysage est un élément de différenciation et d'affirmation d'une identité. Dans ce cadre, les cultures fabriquant des paysages typés deviennent le vecteur d'une identité. Les vignobles, à travers la notion de terroir répondent à ce besoin.

La vision sélective qu'ont les élus de l'agriculture est en accord avec celle des habitants des espaces périurbains qui sont aussi les électeurs des conseils municipaux. Les habitants ont une vision idéalisée de l'agriculture (Donadieu, 1998; Hervieu et Viard, 1996). Ils considèrent la campagne comme un cadre de vie plus qu'un lieu de production. Conformément à cette vision (Fleury et Serrano, 2005), les grandes cultures, symboles d'une agriculture banale, ne sont plus désirées. Le succès de la pétition pour les jardins de Meslay confirme la préférence donnée par les habitants des communes périurbaines à une agriculture de proximité et montre que les élus locaux cherchent à être en accord avec les perceptions de leurs électeurs.

Être le « grenier du reste du monde » prive les collectivités d'une ressource fiscale mais est aussi en dehors de ce qui est considéré par les élus comme un marqueur d'identité ou comme un patri- 
moine local. L'agriculture alimentaire n'est pas un enjeu local, elle relève de décisions nationales voire internationales. En effet, la production alimentaire intensive est standardisée; elle n'est pas un élément de typicité ou de différence. La production s'écoule sur des marchés internationaux qui sont invisibles pour les élus locaux. Les agriculteurs du plateau de Meslay s'orientent vers le modèle beauceron. Ils abandonnent la polyculture-élevage pour se spécialiser dans les grandes cultures. Mais alors que l'agriculture beauceronne est identifiée comme le grenier de la France et peut être à ce titre un élément identitaire pour la Beauce, ce n'est pas le cas pour la Touraine où une autre agriculture joue ce rôle.

\section{OFFRIR DES ZONES D'ACTIVITÉS POUR SE DÉVELOPPER : UN PIÈGE POUR LES COLLECTIVITÉS}

Ouvrir de larges zones économiques et attendre que des entreprises de logistique s'y installent est un pari risqué. En effet, à la surabondance de l'offre foncière locale (Serrano et Demazière, 2009) s'ajoute la concurrence avec d'autres agglomérations pouvant prétendre être une étoile autoroutière et offrir une meilleure articulation entre espace national et espace régional (Davezies, 2007). L'intégration des différentes communautés de communes et d'agglomération devrait, dans un même schéma de coopération territoriale, conduire à une rationalisation du développement économique. Cet objectif du SCoT - en cours depuis 2001 - bute sur des relations entre intercommunalités marquées par la crainte de subir la domination de la ville-centre (Guillard, 2005) et par la capacité des élus locaux à inclure dans les documents supra-communaux à visée stratégique leurs propres projets (Legal et al., 2006). Au final, le SCoT entérinera les projets de développement économique de chaque communauté de communes et se résumera à la somme des projets individuels (fig. 1).

Le périurbain est l'espace privilégié pour l'implantation des entreprises qui se consacrent à la construction, la logistique ou l'énergie. Ces entreprises sont à la recherche de foncier et d'accès routiers rapides. Le foncier et les accès sont des ressources indispensables pour les entreprises mais elles ne sont pas directement génératrices de plus-values; par contre, ces ressources sont contrôlées par les élus et c'est donc vers eux que les entreprises se tournent. Pour les élus des communes périurbaines, il s'agit là de la demande visible des entreprises en quête de possibilités de développement sur leur territoire. Les élus soucieux de favoriser l'implantation d'entreprises sur leurs territoires développent donc des zones d'activités en viabilisant des terrains. Or dans un territoire où les autoroutes sont interconnectées et où le foncier est abondant, l'offre de terrain viabilisé facilement accessible est une ressource banale et répandue dans la plupart des EPCI. Étant en concurrence, les collectivités sont condamnées à surenchérir et à proposer rapidement un grand terrain à une entreprise. Faute de quoi, l'entreprise ira s'installer sur le territoire voisin. Les collectivités ont intérêt à avoir des réserves. Dans ce jeu, les entreprises usent du chantage à la délocalisation faute de capacités d'extension. Les collectivités ont d'autres outils de développement économique que les zones d'activités. En privilégiant l'offre foncière (Serrano et Demazière, 2009) elles estiment rester attractives. Parallèlement, les entreprises ne demandent que du foncier. En l'absence de moyens de réflexion sur leur mode de développement économique, les collectivités périurbaines, ne sont pas incitées à proposer autre chose ou à valoriser autrement leur foncier.

\section{Conclusion}

Bien que le dispositif législatif d'aménagement du territoire ait évolué vers la possibilité de connexions entre politiques agricoles et politiques d'aménagement ce qui permettrait une territorialisation de projets et une prise en compte de la ressource foncière comme ressource territoriale d'intérêt collectif (Jouve et Vianey, 2009) les projets centrés sur la libération de foncier demeurent. La mobilisation des habitants pour les jardins de Meslay a bien obligé les élus à reconsidérer leur perception de l'agriculture. Mais, sous pression, ils ne l'ont fait qu'à la marge. 
Au détriment de la recherche d'autres leviers, lors de la discussion, la maîtrise de l'affectation des sols par les élus conjugue attractivité des espaces périurbains et abondance des réserves foncières. Dans ces débats locaux, la fonction alimentaire est reléguée en arrière-plan. En initiant les changements d'usages des sols, les élus utilisent le foncier comme moyen de développement privilégié; ces changements d'usage sont déterminés par les relations tissées par les différents utilisateurs du sol - qu'ils soient publics ou privés - ce qui revient à accorder la faveur aux intérêts privés. Cette consommation de terres interpelle la possibilité de nourrir une population mondiale envisagée de 9 milliards d'habitants à l'horizon 2050 (Agrimonde, 2010). L'échelle locale ne semble pas permettre d'éviter des projets centrés sur la captation de la rente foncière. «Il est, à notre sens, de la responsabilité de l'État de mettre en œuvre des outils permettant à la collectivité de récupérer les plus-values qu'elle crée en modifiant les droits des sols et qui sont susceptibles de lui permettre de financer des modes d'urbanisme respectueux à la fois des besoins de développement urbain et des espaces agricoles ou naturels existants » (Delattre, Napoléone, 2011).

On peut aussi imaginer un dispositif semblable à celui mis en place pour limiter l'émission de gaz à effets de serre mais appliqué à l'artificialisation des terres agricoles. Les collectivités disposeraient d'un quota de terres à urbaniser. Ces quotas seraient échangeables sur un marché. Un tel système offre l'avantage de la responsabilisation des agents et du maintien de la liberté d'entreprendre. Un volume national de terres urbanisables serait défini et réparti au niveau local. Les collectivités pourraient user tout ou partie de ces droits à urbaniser. Elles peuvent les céder si elles choisissent un développement spatial vertical par rapport à un développement horizontal. La valeur des droits à construire serait reliée à la valeur agronomique des sols. Il serait d'autant plus coûteux d'urbaniser que les sols à artificialiser ont une valeur agronomique élevée.

De notre point de vue, un tel système est préférable à un retour direct de l'État dans la gestion des permis de construire. En effet, cela ne ferait que déplacer le problème. La pression des lobbys au niveau local se déplacerait au niveau national entre des acteurs économiques d'envergure nationale ou internationale et les ministères. L'État confronté à la crise économique et à la nécessité de résorber le chômage serait soumis aux mêmes tentations que les élus locaux. L'État aurait un rôle de régulateur et d'encadrement. Les collectivités conservent la responsabilité du choix de l'usage des sols.

La mise en œuvre d'un système de quotas de sols à artificialiser indexé sur la valeur de production agronomique des sols pose de nombreux problèmes. Tout d'abord, la définition de la valeur agronomique n'est pas aisée. Des sols ingrats pour les céréales peuvent être néanmoins bons pour la viticulture etc. À quel échelon faut-il répartir les droits à construire : commune, établissement public de coopération intercommunale, autre? Faut-il libéraliser totalement les échanges de permis à artificialiser ou mettre des barrières géographiques pour éviter les phénomènes d'évasion et de concentration des droits par rapport à des territoires. Enfin, quelles sont les contreparties échangeables contre les droits à artificialiser si les échanges se font entre les collectivités? Ce système pour complexe qu'il soit, réintroduit la valeur de production dans les négociations entre les acteurs. Il est de nature à modifier l'échelle de valeurs et les hiérarchies implicites décrites précédemment tout en laissant les acteurs libres de faire leurs choix.

\section{Bibliographie}

Abric J.-C., 2003 (rééd. 1994). Pratiques sociales et représentations. Psychologie sociale, Paris, PUF, 252 p.

Agence d'unbanisme de L'agglomération tourangelle, 2006, Synthèse des évolutions des secteurs économiques, Tours, $73 \mathrm{p}$.

AgRIMONDE, 2010. Scénarios et défis pour nourrir le monde en 2050, Paris, Quae, coll. « Matière à débattre et décider ", $259 \mathrm{p}$.

Bonnard M., 2009. Les collectivités territoriales en France, Paris, La documentation française, 152 p.

Chivallon C., 2004. Débattre autour du postmodernisme : commentaires de textes choisis. Espace Géographique, tome $33, n^{\circ} 1 / 2004$, p. 43-58. 
Сoulomb P., 1999. La politique foncière agricole en France, Cahiers Options méditerranéennes, n 36, Politiques foncières et aménagements des structures agricoles dans les pays méditerranéens, p. 69-94.

DelattRe L., Napoléone C., 2011. Écologiser les documents d'urbanisme pour protéger les terres agricoles et les espaces naturels, Courrier de l'environnement de l'INRA, nº 60, p. 63-75.

DDAF 37, 2005. Document de gestion de l'espace agricole et forestier, ministère de l'Agriculture, Paris, CD ROM.

Direction régionale de l'équipement Région Centre, Direction départementale de l'équipement, INSEE, 2001. Référentiel territorial urbain: portrait de l'aire urbaine de Tours, Orléans, INSEE, 71 p.

Davezies L., 2007. L'axe Ligérien : un territoire "métropolitain » en devenir?, Paris, Université Paris 12, 22 p.

Del Casino Jr. V. J., Hanna S. P., Paul Jones III J., 2000. Methodological frameworks for the geography of organizations, Geoforum, n 31, p. 523-538.

Donadieu P., 1998. Campagnes urbaines, Actes Sud, Paris, 219 p.

Duvernoy I., 2002. Espace agricole périurbain et politiques communales d'aménagement : l'exemple de l'agglomération albigeoise, Cybergéo, n 208.

Fleury A., Serrano J., 2005, L'agriculture banale a-t-elle une place dans le projet agriurbain?, L'Harmattan, Le Pradel, p. 243-254.

Guillard C., 2005. Scot et pratiques territoriales, de la théorie à la pratique en Indre-et-Loire, École Polytechnique de l'université de Tours, Tours, 82 p.

Hervieu B., Viard J., 1996. Au bonheur des campagnes (et des provinces), La Tour-d'Aigues, L'Aube, 156 p.

INRA, INSEE, 1998. Les campagnes et leurs villes, Paris, 203 p.

Jouve A.-M., Vianey G., 2009. Le foncier, une ressource territoriale difficile à construire en périurbain, Communication pour les $3^{\text {es }}$ journées de recherches en sciences sociales organisées par l'INRA, la SFER et le CIRAD -Montpellier, CD-ROM.

Legal Y., Mangin D., Wiel M., 2006. La planification spatiale française est-elle dans l'impasse?, Urbanisme, $n^{\circ} 347$, p. 21-23.

Martin S., Bertrand N., Rousier N., 2006. Les documents d'urbanisme, un outil pour la régulation des conflits d'usage de l'espace agricole périurbain ?, Géographie économie société, n 8, p. 329-349.

Ministère de L’Agriculture et de la PÊche, 2001. Recensement agricole 2000 : viticulture la qualité toujours en jeu, Agreste Indre-et-Loire, n 18, p. 4.

Moscovici S., Doise W., 1992. Dissensions et consensus. Une théorie générale des décisions collectives, PUF, Paris, 296 p.

Pointereau P., Coulon F., 2009. Abandon et artificialisation des terres agricoles, Le courrier de l'environnement de l'INRA, n 57, p. 109-120.

Raffestin C., 1986. Territorialité : Concept ou Paradigme de la géographie sociale?, Geographica Helvetica, $\mathrm{n}^{\circ} 2$, p. $91-96$.

Richer M., 2005. Innovacion social y desarollo local en un municipio andino, Cayapa, Revista Venazolana de economia rural, vol. 5, n 9, p. 49-65.

Rouquette M.-L., Rateau P., 1998. Introduction à l'étude des représentations sociales, Grenoble, PUG, 159 p. Roux E., VANier M., 2008. La périurbanisation : problématiques et perspectives (n.8), Paris, DIACT, 88 p.

Santos M., 1990. Espace et méthode, Traduit du brésilien par M.-H. Tiercelin, Paris, Publisud, 123 p.

Serrano J., Demazière C., 2009. Développement économique et gestion de l'espace agricole et naturel, Les tensions au niveau local, le cas de l'agglomération de Tours (France), Territoires Wallons, n 3, p. 123-134.

Cet article a été reçu le 13 février 2011 et définitivement accepté le 2 novembre 2011. 